\title{
Long period variables in 47 Tuc: direct evidence for lost mass
}

\author{
T. Lebzelter ${ }^{1}$ and P. R. Wood ${ }^{2}$
}

\author{
1 Department for Astronomy (IfA), University of Vienna, Türkenschanzstrasse 17, 1180 Vienna, Austria \\ e-mail: lebzelter@astro.univie.ac.at \\ 2 Research School for Astronomy \& Astrophysics, Australian National University, Cotter Road, Weston Creek ACT 2611, Australia
}

Received 18 May 2005 / Accepted 3 July 2005

\begin{abstract}
We have identified 22 new variable red giants in 47 Tuc and determined periods for another 8 previously known variables. All red giants redder than $V-I_{\mathrm{c}}=1.8$ are variable at the limits of our detection threshold, which corresponds to $\delta V \approx 0.1 \mathrm{mag}$. This colour limit corresponds to a luminosity $\log L / L_{\odot}=3.15$ and it is considerably below the tip of the RGB at $\log L / L_{\odot}=3.35$. Linear non-adiabatic models without mass loss on the giant branch can not reproduce the observed PL laws for the low amplitude pulsators. Models that have undergone mass loss do reproduce the observed PL relations and they show that mass loss of the order of $0.3 M_{\odot}$ occurs along the RGB and AGB. The linear pulsation periods do not agree well with the observed periods of the large amplitude Mira variables, which pulsate in the fundamental mode. The solution to this problem appears to be that the nonlinear pulsation periods in these low mass stars are considerably shorter than the linear pulsation periods due to a rearrangement of stellar structure caused by the pulsation. Both observations and theory show that stars evolve up the RGB and first part of the AGB pulsating in low order overtone modes, then switch to fundamental mode at high luminosities.
\end{abstract}

Key words. stars: late-type - stars: AGB and post-AGB - stars: oscillations - stars: mass-loss

\section{Introduction}

It is now well established that pulsating red giant stars lie on a series of up to six parallel period-luminosity (PL) sequences (e.g. Wood et al. 1999; Wood 2000; Ita et al. 2004; Soszyński et al. 2004; Fraser et al. 2005). The stars known to populate these sequences are generally field variables so that their metallicities, masses and ages are not known individually, although the luminosities are well known because the stars lie in stellar systems at a known distance, such as the SMC and LMC. The dispersion in the PL relations is much larger than the amplitude of the pulsation, especially for the small amplitude variables, and this dispersion is almost certainly a consequence of the dispersion in mass and metallicity at a given pulsation period.

Comparison of the PL relations with theory (Wood et al. 1999) shows that four of the PL sequences (sequences $\mathrm{A}, \mathrm{B}$ and C of Wood et al. 1999, or sequences A, B, C and $C^{\prime}$ of Ita et al. 2004) can be broadly explained by radial pulsation in the lower order modes. However, a real test of the theoretical models requires comparison with stars of known metallicity, age and (initial) mass since the fits of theory to data in Wood et al. (1999) and Ita et al. (2004) are not particularly good.

Globular clusters are well suited for carrying out such a comparison, and for studying the relation between pulsation, mass loss and stellar evolution along the giant branch, since fundamental parameters like initial mass, luminosity and metallicity are well known. However, only a small number of pulsating red giants, hereinafter referred to as long-period variables or LPVs, is known in any given globular cluster (Clement et al. 2001), with 47 Tuc having the most known LPVs (14). This is an insufficient number of variables for clearly defining PL sequences, especially as many of the periods of the LPVs are poorly determined. The origin of this problem is that most search programs for variable stars in globular clusters were designed to optimize detection and period determination for variables with periods of about one day or less. LPVs with their periods of a few ten to a few hundred days have therefore not been well surveyed and the existing samples are far from complete.

To rectify this situation, we have started a search program for long period variables in Galactic Globular clusters (Lebzelter et al. 2004). The relatively large number of already known or proposed long period variables in 47 Tuc made it a good starting point. Here, we present the results for 47 Tuc (NGC 104), the first cluster analyzed. We adopt the following properties for 47 Tuc: $(m-M)_{V}=13.5 \pm 0.08$ (Gratton et al. 2003); metallicity $[\mathrm{Fe} / \mathrm{H}]=-0.66$ (Carretta \& Gratton 1997); interstellar reddening $E(B-V)=0.024$; an age of $11.2 \pm 1.1 \mathrm{Gyr}$ (Gratton et al.2003); and a turnoff mass between 0.86 and $0.9 M_{\odot}$. In a recent paper discussing radial velocity variations in known LPVs in 47 Tuc (Lebzelter et al. 2005) we gave more discussion of the properties of 47 Tuc and its variables and we refer the reader to that paper for details. 


\section{Observations}

\subsection{Mount Stromlo data}

Our monitoring program of 47 Tuc started in August 2002 at the 50 inch telescope at Mount Stromlo. This telescope was equipped with a two channel camera used earlier for the MACHO experiment (Alcock et al.1992). The camera obtained two images in two broad band ranges at the same time (Marshall 1994). These passbands did not correspond to standard filters but the blue one had a mean wavelength similar to Johnson $V$. The camera covers a field of about 0.5 square degree on the sky with a pixel scale of 0.62 arcsec. The whole cluster could thus be observed with one observation, and most of the cluster was on one CCD of the MACHO camera (there are four CCDs per filter band). Observations were obtained once to twice a week. A few frames were lost due to technical problems or bad seeing conditions. Our monitoring came to an early end after about five months when Mount Stromlo Observatory was destroyed by a bush fire. Altogether we collected 15 useable frames over this time span. All observations were done in queue observing mode.

For the determination of light curves and the detection of variables, only the blue frames were used. The light amplitude of long period variables is typically larger in the blue than in the red (e.g. Fox 1982) making the detection of variables and the determination of periods easier. Furthermore, many bright stars on the red frame were over-exposed and thus not useable. The blue detector had an area of bad pixels as well as a few scattered dead pixels. Consequently, due to small positional shifts between the different observing nights some stars could not be measured on all 15 frames.

\subsection{CTIO data}

From August 2003 to January 2004, we continued the monitoring with ANDICAM at CTIO's $1.3 \mathrm{~m}$ telescope operated by the SMARTS consortium. The size of the CCD field is $6 \times 6$ arcmin and the pixel scale is 0.37 arcsec/pixel. A description of the camera is given in DePoy et al. (2003). Due to the smaller FOV we had to make a mosaic of images to cover most of the cluster. Even then, we were limited to the central part, and some of the outer areas covered by the Mount Stromlo data could not be observed. Observations were done in $V$ and $I_{\mathrm{c}}$. Observations were scheduled roughly once per week giving a total of 16 useable frames. As for the Mount Stromlo data, observations were done in queue observing mode.

\subsection{Other data}

Six observations of the cluster in $V$ and $I_{\mathrm{c}}$ were taken in service mode with the WFI at the ESO $2.2 \mathrm{~m}$. These observations were obtained between June and September 2002. Four additional observations taken in July/August 2003 were kindly provided by Laszlo Kiss and collaborators. These observations in $V$ and $I_{\mathrm{c}}$ were obtained with the Siding Spring Observatory 40-inch telescope, helping to reduce the gap between the Mount Stromlo and the CTIO data set.
For a different project (Lebzelter et al.2005), we did a short photometric monitoring of some parts of 47 Tuc with ANDICAM at CTIO's $1.3 \mathrm{~m}$ telescope in March to May 2002. These data were obtained in the $V$-filter with the old ANDICAM CCD which had severe limitations (only half of the chip was useable). These data were used only in rare cases.

\section{Data reduction}

For the Mount Stromlo and the CTIO data, flatfield and bias correction was done as part of the standard data pipeline. The 40inch data were reduced applying standard data reduction with MIDAS, while the WFI data were reduced with the corresponding IRAF package. For the detection of variables and the measurement of the light curves on the Mount Stromlo and CTIO frames we used the image subtraction code ISIS 2.1 by Alard (2000). First, the two data sets were analyzed separately. The reference flux of the identified variables required to produce light curves from the image subtraction measurements was derived using the PSF fitting software written by Ch. Alard for the DENIS project. A description of this code can be found in Schuller et al. (2003). For the photometric calibration of the CTIO data we used standard stars from Landolt's field RU 149 (Landolt 1992). Photometric accuracy of the resulting light curves could be analyzed by comparing measurements of variables in overlapping parts of the CTIO mosaic. Typical deviations were of the order of $0.007 \mathrm{mag}$. We then used about 40 non-variable cluster stars ranging in $V-I$ between 0.9 and 2.1 to link the Mount Stromlo measurements to those from CTIO. Typical errors in the Mount Stromlo magnitudes were of the order of $0.013 \mathrm{mag}$.

For various reasons (field of view, pixel scale, damaged parts of the $\mathrm{CCD}$, depth of the observation) not all variables could be measured on both the CTIO and the Mount Stromlo data. In these cases only one of the data sets could be used for the analysis.

Our aim was to detect and measure long period variables on the upper part of the giant branch only. Thus we selected for analysis only those variables that varied on time scales of more than approximately 30 days with a total light amplitude of at least $0.1 \mathrm{mag}$ in $V$ (or the blue MACHO pass band). For stars with shorter periods, our sampling was not sufficient to estimate a useful period. We searched for periods in the selected light curves using the Fourier analysis code Period98 (Sperl 1998). A maximum of two periods was derived for each star. First, the two data sets from Mount Stromlo and CTIO were analyzed separately. The observations from ESO and SSO, measured using PSF fitting, were inserted in the combined light curves. Then a period search was done on the whole light curve. Generally, the agreement between the periods derived from these three data sets agreed very well. Naturally, stars with periods exceeding the length of one of the data sets or with high irregularity in their light curve lead to deviating results. The uncertainties of the periods calculated from a least square fit to the complete data sets are in most cases less than a few percent. Of course the semiregular nature of most of the stars in our sample means that the derived period is representative only 
for the current variability behaviour. For the long period cases, we used only the period derived from the combined light curve. Some stars were classified as irregular if no consistent period could be found. These stars will not be discussed further in this paper.

\section{The variables}

Periods have previously been published in the literature for the LPVs V1-V8, V11, V13, V18 and V21 (we use the nomenclature of Clement et al.2001). Light curves for these stars derived from the current observations are given in Lebzelter et al. (2005), along with some discussion of the origin and reliability of the published periods. These periods are given in Col. 7 of Table 1. Beside these stars, a few more red giants have been identified as variables before, namely V15, V16, V17, V19, V20, V22, V23, V25, V27, V28 and A19. For some of these (V15 to V17, V25 and V28) periods were given by Fox (1982). A19 was reported variable by Lloyd-Evans (1974), but no period was given. Later variability surveys, like those of Kaluzny et al. (1998) and Weldrake et al. (2004), have focused on binaries and RR Lyr stars and thus typically were not suitable for determining periods of the stars on the upper giant branch due to overexposure. The long period variables they report are most likely members of the Small Magellanic Cloud.

Table 1 lists the variables detected or characterized in the course of this study. Our sample includes 22 variables detected for the first time, and we give the first period determinations for six further stars previously known to be variable. All these periods are listed in Col. 6 of Table 1. We do not include in Table 1 any previously known variables that we were not able to monitor (because they fell on CCD defects or outside our field of view). As nomenclature for new variables, we use LWxx with the numbering going from east to west. Near infrared $J$ and $K$ magnitudes were extracted from the 2MASS database and they are listed in Cols. 4 and 5. The 2MASS coordinates are given in Cols. 2 and 3.

Light variation versus time are presented in Fig. 1, together with the fits from Fourier analysis. For selected variables with a reliable period determination and no long secondary period we show the light curve plotted against phase in Fig. 2. In Fig. 1 obvious deviations of the data from the fit illustrate the semiregular nature of many stars of the sample. It can be seen that quite a large fraction of variables shows a long secondary period lasting several hundred to several thousand days. As these periods exceed the length of the time span monitored, we could not determine their length accurately and thus we do not give them in Table 1. They are included in the fits for illustrative purposes only. The formal ratio between the long and the short period in the plot ranges between 4 (LW4) and 100 (LW15). In most cases the second period is 10 or more times longer than the shorter period.

The Fourier fits to the stars V20, LW10, LW11, LW12 and LW13 deserve special comment. V20 and LW10 both appear to have periods of about 220-230 days but, because of the time gap in our observations, these periods are quite uncertain. Figs. 1 and 2 suggest that these two stars have light curves with bumps during rising light, a feature found in the light curves of several miras in the solar neighbourhood. For LW10 the semiperiod shows up clearly in the Fourier analysis although this is not the case for V20. The fit of LW11 is rather bad and the period determined is very uncertain. In LW12, the period and amplitude of the light curve clearly changed between the Mount Stromlo and CTIO observations and we use separate Fourier fits to the two sets of observations. The Mount Stromlo data set gave a period of $61 \mathrm{~d}$ while the CTIO data give $116 \mathrm{~d}$. The fit to LW13 is poor, and the plotted fit curves include a phase change of 20 days between the Mount Stromlo and CTIO observations. Finally, we note that the 2 MASS observations could not separate LW13 from its close companion.

Multiperiodicity is a well known phenomenon among red variables in the solar neighbourhood (e.g. Percy et al.2003; Kiss et al.1999). Long secondary periods are well known among long period variables, but a physical explanation for them has not yet been found (Wood et al. 2004).

In Figure 3 we show the position of the variables in the colour-magnitude diagram using magnitudes from the CTIO images or, for a few stars, an alternative source given below. Only the giant branches and the horizontal branch are shown. Beside the variables listed in Table 1, we also marked stars detected as variables but slightly below our amplitude cutoff criterion and stars with large irregularities in the light curve so that a period could not be determined. All $V$ and $V-I_{\mathrm{c}}$ values of the variables are mean values derived from our light curves. For V5, V7, V11, V13 and V18 no CTIO data have been obtained. Instead, we give the values from Fox (1982). V3 was also not observed at CTIO, but $V$ and $I_{K}$ light curves were obtained by Eggen (1975) and we use the mean magnitudes from his observations, converting $V-I_{K}$ values to $V-I_{\mathrm{c}}$ using the transform in Bessell (1979). V2 and LW10 are missing because the stars were saturated or unmeasurable on some of our $I$ frames.

Figure 3 illustrates that all variables in our sample, with the possible exception of V19, are almost certainly on the upper giant branch of 47 Tuc (rather than in the SMC or Galactic halo). V19 is located above and to the left of the 47 Tuc AGB. There is no indication for a long secondary period in this star (see Fig. 1) which may lead to a non-representative measurement ${ }^{1}$. At the blue end of the giant branch where V19 is found, its amplitude of more than $0.8 \mathrm{mag}$ is rather untypical. Possible explanations may be that it has a very close blue neighbour that was not separated from V19 in our images, or it may be a star in the Galactic halo.

The most interesting aspect of Fig. 3 is that it clearly shows that all stars on the giant branch of 47 Tuc redder than $V-I_{\mathrm{c}} \approx 1.8$ are variable.

Finally, we comment on the connection between LPV pulsation and mass loss in 47 Tuc. Origlia et al. (2002) listed five stars in 47 Tuc with a high infrared excess, indicating that they are surrounded by some dust. All five are located within our investigated field. Identification is not simple as Origlia et al. provide only near infrared finding charts of low spatial

\footnotetext{
1 Long secondary periods may be responsible for some of the scatter along the giant branch since the mean brightness is calculated over only part of the light cycle corresponding to these periods.
} 
Table 1. The LPVs of 47 Tuc.

\begin{tabular}{|c|c|c|c|c|c|c|c|c|c|}
\hline Name & $\alpha(2000)$ & $\overline{\delta(2000)}$ & $\overline{\bar{V}}$ & 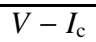 & $\overline{J J}$ & $\overline{\bar{K}}$ & $\overline{P[\mathrm{~d}]}$ & $P_{\text {literature }}$ & Remark \\
\hline V1 & 002412.4 & -720639 & 13.15 & 3.86 & 7.45 & 6.21 & 221 & 212 & 1 \\
\hline V2 & 002418.4 & -720759 & - & - & 7.52 & 6.29 & 203 & 203 & 1,2 \\
\hline V3 & 002515.9 & -720354 & 12.63 & 3.54 & 7.49 & 6.27 & 192 & 192 & 1 \\
\hline V4 & 002400.3 & -720726 & 12.34 & 2.62 & 7.87 & 6.69 & 165 & 82,165 & 1,3 \\
\hline V5 & 002503.7 & -720931 & 11.80 & 1.99 & 8.65 & 7.47 & 50 & 60 & 1,4 \\
\hline V6 & 002425.5 & -720630 & 11.74 & 1.94 & 8.54 & 7.43 & 48 & 48 & 1 \\
\hline V7 & 002520.6 & -720640 & 11.83 & 2.30 & 8.18 & 6.97 & 52 & 52 & 1,4 \\
\hline V8 & 002408.3 & -720354 & 12.01 & 2.39 & 7.94 & 6.70 & 155 & 155 & 1 \\
\hline V11 & 002509.0 & -720217 & 12.03 & 2.70 & 7.91 & 6.71 & 160: & 52,100 & 4,5 \\
\hline V13 & 002258.3 & -720656 & 12.36 & 2.23 & 8.79 & 7.70 & $40+$ long & 40 & $1,4,8$ \\
\hline V16 & 002523.2 & -721105 & 11.65 & 2.0 & 8.35 & 7.23 & 41, 88: & 45 & \\
\hline V18 & 002509.2 & -720239 & 11.67 & 1.95 & 8.59 & 7.47 & 83: & & 1,4 \\
\hline V19 & 002414.8 & -720444 & 11.46 & 1.70 & 8.79 & 7.61 & 83 & & \\
\hline V20 & 002414.5 & -720509 & 12.30 & 2.53 & 8.08 & 6.94 & 232: & & 11 \\
\hline V21 & 002350.1 & -720550 & 12.41 & 2.85 & 8.07 & 6.78 & $76:$ + long & & 1 \\
\hline V22 & 002408.9 & -720300 & 11.80 & 1.99 & 8.38 & 7.18 & 62 & & 8 \\
\hline V23 & 002429.5 & -720908 & 11.77 & 2.08 & 8.68 & 7.53 & $52+$ long & & \\
\hline V25 & 002358.9 & -720235 & 11.96 & 2.50 & 8.20 & 7.03 & 44 & 42 & 9 \\
\hline V27 & 002415.2 & -720436 & 12.11 & 2.52 & 7.97 & 6.77 & $69+$ long & & \\
\hline A19 & 002421.8 & -720413 & 12.18 & 2.57 & 8.08 & 6.79 & 60 & & \\
\hline LW1 & 002322.3 & -720540 & 11.83 & 2.08 & 8.39 & 7.19 & 39 & & 9 \\
\hline LW2 & 002329.2 & -720620 & 11.90 & 2.23 & 8.26 & 7.05 & $60+$ long & & 9 \\
\hline LW3 & 002347.4 & -720653 & 11.92 & 2.35 & 8.13 & 6.89 & 107 & & \\
\hline LW4 & 002351.3 & -720349 & 11.80 & 1.98 & 8.56 & 7.37 & 32 + long: & & 9 \\
\hline LW5 & 002353.2 & -720416 & 11.74 & 1.96 & 8.53 & 7.32 & 40 & & \\
\hline LW6 & 002354.7 & -720339 & 11.85 & 2.00 & 8.51 & 7.30 & 41 & & 9 \\
\hline LW7 & 002356.9 & -720533 & 11.66 & 1.86 & 8.26 & 7.18 & 42 & & 10 \\
\hline LW8 & 002357.7 & -720530 & 11.82 & 1.88 & 8.10 & 7.12 & $27:+$ long & & 9 \\
\hline LW9 & 002358.2 & -720549 & 12.34 & 2.77 & 7.97 & 6.74 & 74 & & 9 \\
\hline LW10 & 002402.6 & -720507 & 12.22 & - & 7.57 & 6.40 & 110:, 221: & & $2,10,11$ \\
\hline LW11 & 002403.2 & -720451 & 11.95 & 1.79 & 8.47 & 7.40 & 36: & & 11 \\
\hline LW12 & 002404.0 & -720510 & 11.96 & 2.35 & 8.12 & 6.89 & 61,116 & & 11 \\
\hline LW13 & 0024 07.9: & -7204 32: & 12.56 & 2.93 & 7.44: & $6.25:$ & 65: & & 6,11 \\
\hline LW14 & 0024 09.4: & $-720449:$ & 11.71 & 1.99 & 8.42 & 7.39 & 50 & & 7,10 \\
\hline LW15 & 002411.2 & -720509 & 11.76 & 2.05 & 8.32 & 7.13 & $46+$ long & & 9 \\
\hline LW16 & 002413.6 & -720452 & 11.88 & 1.89 & 8.39 & 7.32 & $29+$ long & & 9 \\
\hline LW17 & 002416.3 & -720131 & 11.81 & 1.99 & 8.43 & 7.20 & 41 & & 9 \\
\hline LW18 & 002420.5 & -720450 & 11.91 & 2.33 & 7.95 & 6.77 & $65+$ long & & \\
\hline LW19 & 002423.2 & -720423 & 11.85 & 2.17 & 8.38 & 7.16 & $40+$ long & & 9,10 \\
\hline LW20 & 002452.1 & -715611 & - & - & 8.31 & 7.11 & 49 & & 2,8 \\
\hline LW21 & 002523.2 & -721105 & - & - & 8.44 & 7.28 & 38 & & 2,8 \\
\hline LW22 & 002530.1 & -720432 & 12.06 & 2.48 & 8.16 & 6.93 & $63+$ long & & \\
\hline
\end{tabular}

Notes: Cols. 2 and 3 give coordinates from 2MASS. Column 4 is the mean $V$ brightness. $J$ and $K$ colours are from 2MASS converted to the AAO system, if not stated otherwise. If there is an indication for a long secondary period exceeding the time span of the monitoring, the star is marked with "+long". Uncertain values are marked with a colon.

Remarks: (1) see Lebzelter et al. (2005); (2) no $V$ or $V-I_{\mathrm{c}}$, not in the CMD (Fig. 1); (3) currently only the longer period is visible; (4) photometry in the CMD from Fox (1982) - see text; (5) current periodicity unclear, see Lebzelter et al. (2005) for details; (6) close neighbour on 2MASS images, NIR fluxes and position possibly wrong; (7) not in 2MASS point source catalogue, coordinates calculated from our images, $K$ magnitude from Origlia et al. (2002); (8) only on Mount Stromlo frames; (9) no Mount Stromlo data; (10) stars with near infrared excess detected by Origlia et al. (2002); (11) see text in Sect. 4 for comments on the periods.

resolution. Three stars from their list can be identified unequivocally with optical counterparts (stars number 1,3 and 5 from Origlia et al.). All three are variables, namely LW10, LW14 and LW19, respectively. The source number 4 from Origlia et al. is probably related to the variable star LW7. The fifth star listed in Origlia et al. (their number 2) could not be identified with any
LPV. It is the star with the weakest infrared excess in their list. It is surprising that the star (LW19) with the largest mass loss rate in Origlia's list (number 5) is neither one of the brightest variables nor has it a long period. A similar comment can be made about V18, the variable with the largest infrared excess in a survey of 47 Tuc by Ramdani \& Jorissen (2001, see also 

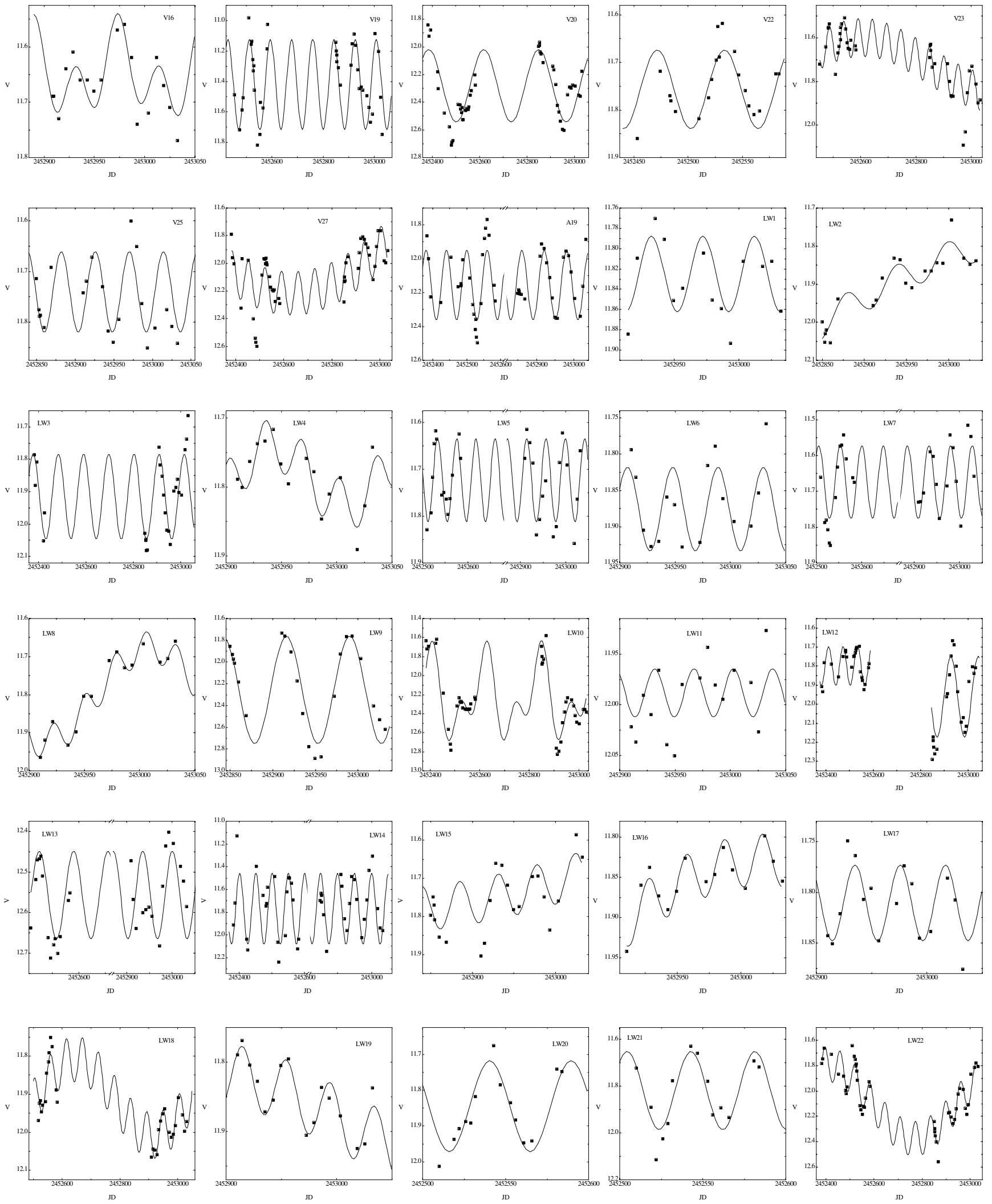

Fig. 1. Light curves of the variables of our survey, except those stars already discussed in Lebzelter et al. (2005). Fourier fits with up to two periods are shown. Note that both axis scales vary from star to star. For A19, LW5, LW7, LW13 and LW14, a break has been introduced into the time axis for a better presentation of the light change. Errors on data points before JD2452700 are estimated to be 0.013 mag (Mount Stromlo data) while after this date the errors are estimated to be 0.007 mag (CTIO data). 

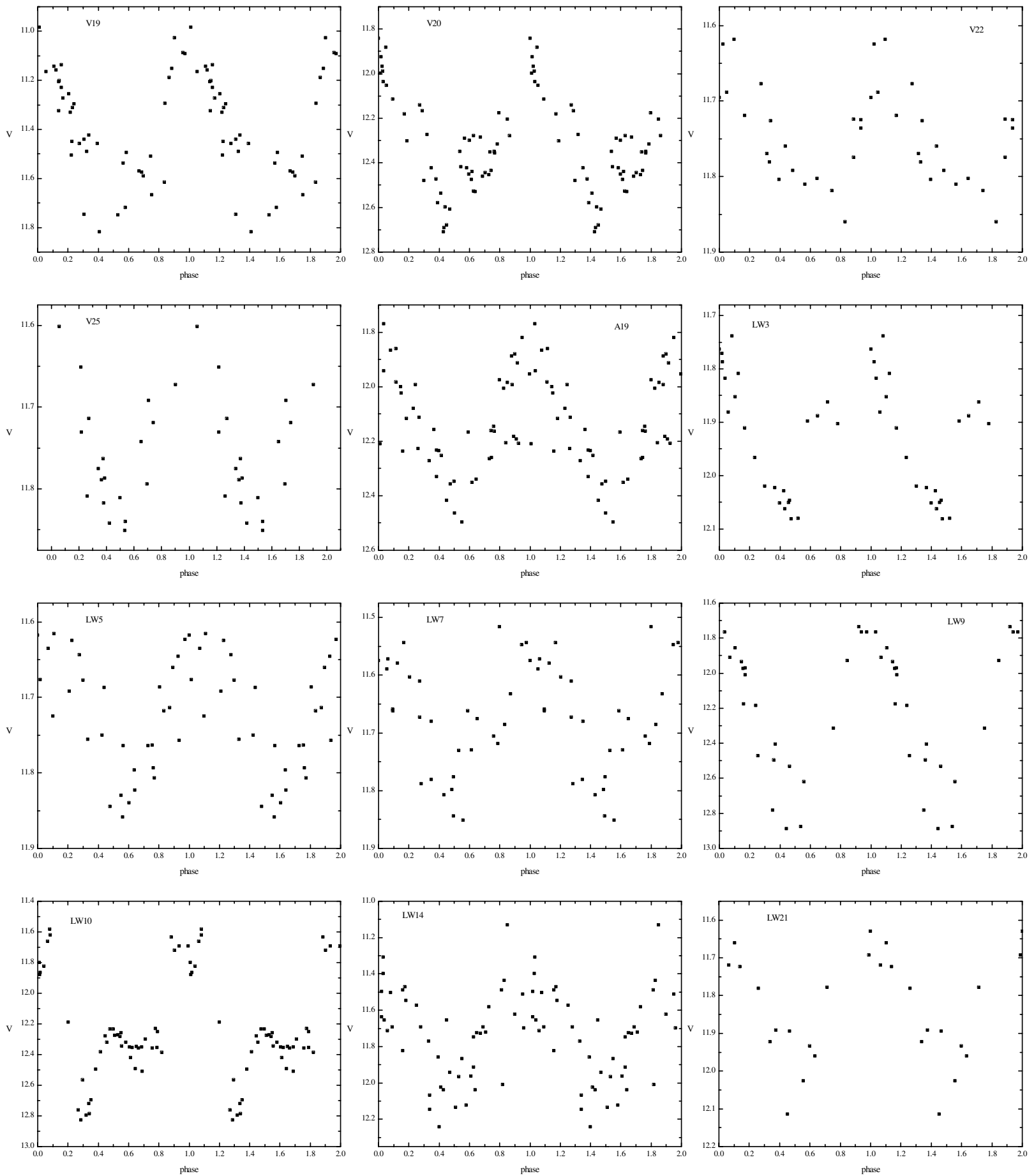

Fig. 2. Light curves versus phases for selected, monoperiodic variables from Table 1 . The period given in the table was used to calculate the phase.

Lebzelter et al. 2005). There is obviously no strict correlation between pulsation period and mass loss rate.

\section{The $K-\log P$ diagram}

Given the large number of LPVs now known in 47 Tuc, we are now in a position to examine the PL relations in the cluster. We do this in the form of the $K-\log P$ relation, which is shown in Fig. 4. Generally, we do not have $K$ light curves for the LPVs in 47 Tuc so we can not readily calculate mean $K$ magnitudes. However, a small number of the variables have been observed many times (see Fox 1982; Menzies \& Whitelock 1985 and Frogel et al. 1981) and good estimates of the mean $K$ magnitude and $K$ amplitude can be obtained. For each star plotted in Fig. 4, the $K$ magnitude is the mean of the maximum and minimum observed $K$ magnitude. For stars without multiple observations, the 2MASS $K$ magnitude has been used. All $J$ and $K$ magnitudes have been converted to the AAO system 


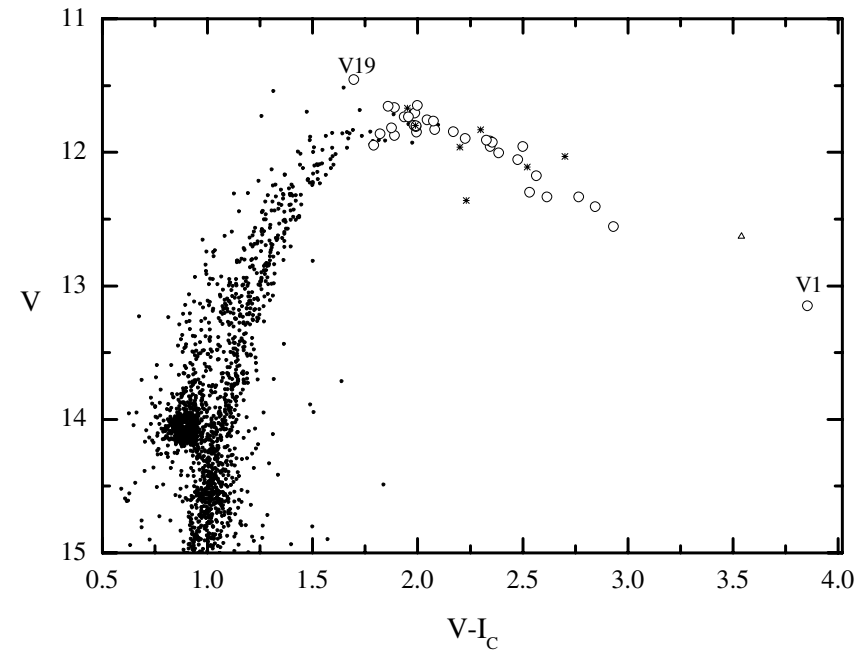

Fig. 3. Colour-magnitude diagram of 47 Tuc. Variable stars are indicated by open circles. Asterisks mark stars taken from Fox (1982) while the triangle is V3 using data from Eggen (1975). For all variables mean magnitudes and colours were used.

using the conversions in Allen \& Cragg (2001) and Carpenter (2001): these are the values shown in Table 1. A comparison of the $K$ amplitude with the $V$ light amplitude obtained in the present study shows that the $K$ amplitude is approximately $20 \%$ of the $V$ amplitude. The error bars in Figure 4 have a full length of $20 \%$ of the full visual amplitude of the pulsation mode associated with each point. Hence, they should represent the $K$ amplitude of the variables.

Also shown in Fig. 4 are the sequences of LPVs in the LMC as given by Ita et al. (2004). The magnitudes have been adjusted to bring them from an LMC distance modulus of 18.55 to a 47 Tuc distance modulus of 13.50. The large-amplitude Mira variables fall close to sequence $C$, while the bulk of the smaller amplitude variables fall near, but not necessarily on, sequences $\mathrm{B}^{+}, \mathrm{B}^{-}$and $\mathrm{C}^{\prime}$. The lack of variables on sequences $\mathrm{A}^{+}$ and $\mathrm{A}^{-}$may be real or it may be that these stars have amplitudes that are below our detection limit of $\sim 0.1 \mathrm{mag}$.

It is notable in Fig. 4 that the 47 Tuc LPVs do not appear to fall exactly on the LMC sequences. For example, the majority of the smaller amplitude variables brighter than $K=7$ fall between sequences $\mathrm{B}^{+}$and $\mathrm{C}^{\prime}$ while fainter than $K=7$, most variables fall between sequences $\mathrm{A}^{-}$and $\mathrm{B}^{-}$with a small number appearing to fall on sequence $\mathrm{C}^{\prime}$. This is probably a result of the different masses of the stars in the 47 Tuc and LMC samples. We address this possibility in the next section.

If we neglect V19 which appears anomalously blue (see Sect. 4), stars seem to evolve up to $K \approx 6.7$ in the 47 Tuc equivalents of sequences $\mathrm{B}$ and $\mathrm{C}^{\prime}$, and to then switch to sequence $\mathrm{C}$ for the final stage of evolution. Using the results in Wood et al. (1999), this switch seems to correspond to a transition in pulsation from a low order radial overtone mode to the fundamental radial mode (compare also Lebzelter et al. 2005).

The tip of the RGB in the $K-\log P$ diagram occurs at $K=7.1$ (see Fig. 6) at $\log L / L_{\odot}=3.355$. From Figs. 4 and 5 we can say that about half of the variables are more luminous

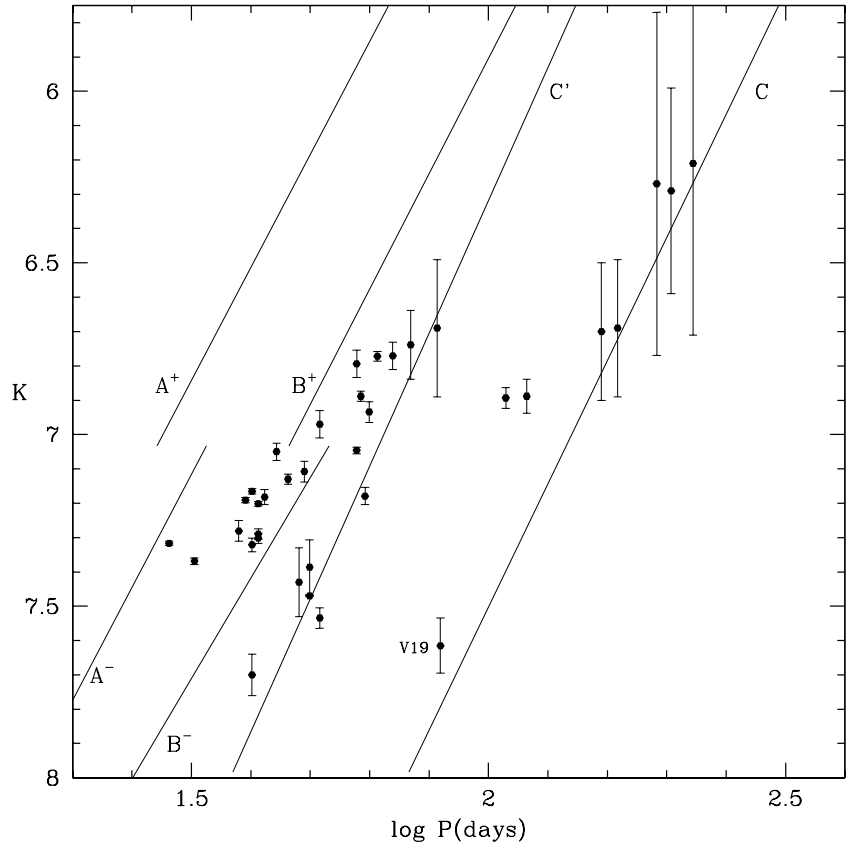

Fig. 4. The $K-\log P$ diagram for the LPVs in 47 Tuc. The error bars are scaled $V$ light amplitudes. Only variables with reasonably welldetermined periods (periods without a following colon in Table 1) are shown. The lines show the sequences of LPVs in the LMC as given by Ita et al. (2004). Sequence $C$ is thought to be linked to fundamental mode pulsation, the other sequences to first to third overtone pulsation.

than the RGB tip and therefore must be AGB stars. The stars below the RGB tip could be either on the AGB or the RGB.

\section{Pulsation models}

\subsection{Description of the models}

With a substantial set of LPVs now known in 47 Tuc, we are in a position to make theoretical models for the periodluminosity laws. For our models, we adopt the following parameters for 47 Tuc (see the Introduction): distance modulus $(m-M)_{V}=13.5$, reddening $E(B-V)=0.024$, helium mass fraction $Y=0.27$, metal abundance $Z=0.004$ and mainsequence turnoff mass $0.9 M_{\odot}$. Since we are computing pulsation periods, it is important to get the radii (and hence $T_{\text {eff }}$ ) of the models correct. In order to realize this condition, the mixing-length in the convection theory was set so that the models coincided with the giant branch of 47 Tuc. Figure 5 shows the models in the HR-diagram. The giant branch of the models clearly passes through the region of the giant branch occupied by the variables.

The properties of the models are given in Table 2 . The linear non-adiabatic pulsation models were created with the pulsation code described in Fox \& Wood (1982), updated to include interior opacities of Iglesias \& Rogers (1993) and low temperature opacities of Alexander \& Ferguson (1994). This code uses a mixing length theory of convection that explicitly treats variation of the convective velocity with time. The core mass $M_{\mathrm{c}}$ was obtained from the $L-M_{\mathrm{c}}$ relation of Boothroyd \& Sackmann (1988). The most luminous RGB model 


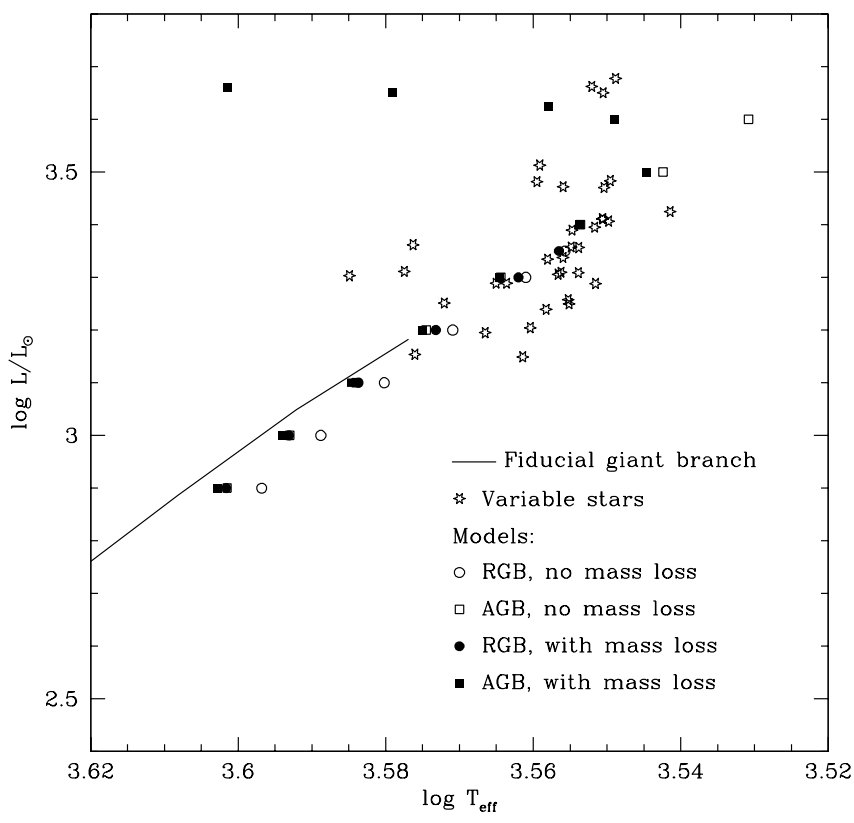

Fig. 5. The tip of the 47 Tuc giant branch in the HR-diagram. $K$ and $J-K$ of the variables were converted to $\log L / L_{\odot}$ and $\log T_{\text {eff }}$ using the transforms in Houdashelt et al. (2000a,b). The fiducial giant branch given by Hesser et al. (1987) is also shown, with $V$ and $B-V$ also converted to $\log L / L_{\odot}$ and $\log T_{\text {eff }}$ using the transforms in Houdashelt et al. The pulsation models are plotted using symbols identified on the figure.

corresponds to the RGB tip luminosity of the $0.9 M_{\odot}$, $Z=0.004$ tracks of Fagotto et al. (1994).

The models with mass loss were constructed according to the following prescriptions. Firstly, note that without mass loss the giant branch should extend to very high luminosities well beyond $\log L / L_{\odot}=10^{4} L / L_{\odot}$. Since the most luminous stars on the 47 Tuc giant branch have $L / L_{\odot} \approx 4000 L / L_{\odot}$, it is assumed that mass loss dissipates the hydrogen-rich envelope at about this luminosity. Evolutionary models with mass loss were constructed adopting a Reimers' mass loss law (Reimers 1975), and the evolution rates on the the giant branch given by the evolutionary tracks of Fagotto et al. (1994). The Reimers mass loss rate was multiplied by a factor $\eta$ which was adjusted so that the stars left the AGB at $L / L_{\odot} \approx 4000 L / L_{\odot}$ (a value $\eta=0.33$ was required). The resulting models are shown in the HR-diagram Fig. 5 and the masses of the models are given in Table 2.

The $K-\log P$ diagram for models both with and without mass loss is compared with the observed $K-\log P$ sequences in Fig. 6. We now consider the small and large amplitude sequences separately.

\subsection{Small amplitude variables}

It is clear that the models without mass loss fail to reproduce the observed periods for the smaller amplitude pulsators (the overtone pulsators, which have $\log P<2$ ). In fact the model sequences avoid the observed sequences. Since the model calculations are for linear pulsation models, they should fit the small amplitude variable sequences.

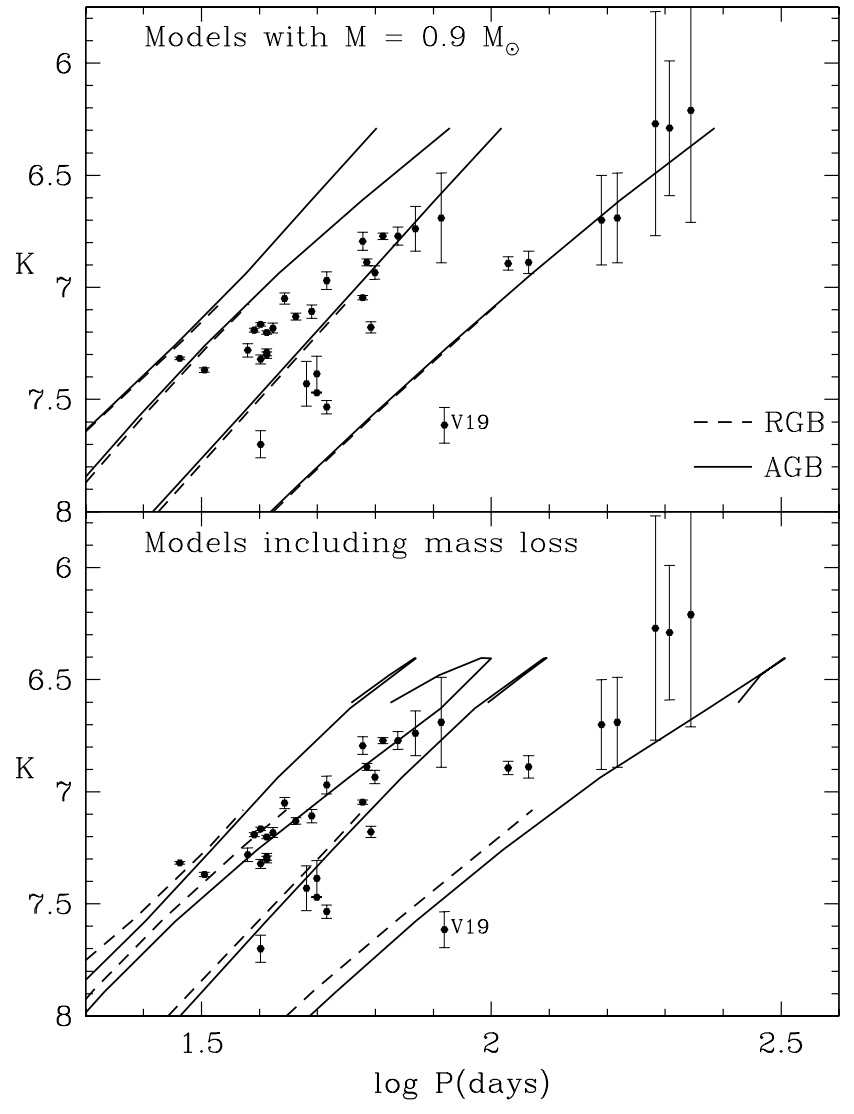

Fig. 6. The $K-\log P$ diagram for models both with and without mass loss. The fundamental mode and the first three overtones are shown. The variables with reasonably well-determined periods are also shown, as in Fig. 4.

A simple way to get the observed and model sequences to agree would be to make the giant branch cooler at a given luminosity. Since the pulsation period for the overtones $P \propto R^{\frac{3}{2}}$ (Fox \& Wood 1982), and since $L=4 \pi \sigma R^{2} T_{\text {eff }}^{4}$, at a given luminosity $P \propto T_{\mathrm{eff}}^{-3}$. In order to fit the theoretical overtone sequences to the observed sequences, $\log P$ needs to increase by $\sim 0.15$, which means that $\log T_{\text {eff }}$ needs to decrease by $\sim 0.05$. As can be seen from Fig. 5, this is far greater than any likely uncertainty in $\log T_{\text {eff. }}$. We therefore conclude that models that have not undergone considerable mass loss since the mainsequence can not explain the pulsation periods of the overtone LPVs in 47 Tuc.

In contrast to the models without mass loss, the overtone pulsation periods for the stars that have undergone mass loss agree well with the observations. The periods of the mass loss models are longer than those of the models without mass loss due to the lower stellar mass $\left(P \propto M^{-\frac{1}{2}}\right)$, and the slope of the $K-\log P$ relations is smaller due to the decrease in mass with luminosity. The decreased slope is particularly prominent for the second overtone which moves from having a period close to that of the 3rd overtone at low luminosities to a period close to that of the 1 st overtone at high luminosities. 
Table 2. The pulsation models.

\begin{tabular}{|c|c|c|c|c|c|c|c|c|}
\hline$L / L_{\odot}$ & $M / M_{\odot}$ & $M_{\mathrm{c}} / M_{\odot}$ & $\ell / H_{\mathrm{p}}$ & $\log T_{\text {eff }}$ & $P_{0}$ & $P_{1}$ & $P_{2}$ & $P_{3}$ \\
\hline \multicolumn{9}{|c|}{ RGB - no mass loss } \\
\hline 794 & 0.9000 & 0.4002 & 1.80 & 3.5987 & 27.6 & 18.1 & 12.5 & 9.6 \\
\hline 1000 & 0.9000 & 0.4136 & 1.80 & 3.5906 & 36.1 & 23.2 & 15.8 & 12.3 \\
\hline 1259 & 0.9000 & 0.4274 & 1.80 & 3.5818 & 47.7 & 29.8 & 20.1 & 16.2 \\
\hline 1585 & 0.9000 & 0.4417 & 1.80 & 3.5724 & 64.0 & 38.2 & 25.6 & 21.8 \\
\hline 1995 & 0.9000 & 0.4565 & 1.80 & 3.5621 & 87.3 & 49.2 & 33.1 & 29.4 \\
\hline 2239 & 0.9000 & 0.4640 & 1.80 & 3.5567 & 102.6 & 56.0 & 38.2 & 34.0 \\
\hline \multicolumn{9}{|c|}{ AGB - no mass loss } \\
\hline 794 & 0.9000 & 0.4737 & 1.80 & 3.6034 & 26.5 & 17.3 & 11.9 & 9.2 \\
\hline 1000 & 0.9000 & 0.4783 & 1.80 & 3.5948 & 34.8 & 22.2 & 15.2 & 11.8 \\
\hline 1259 & 0.9000 & 0.48 & 1.80 & 3.5857 & 46.2 & 28.6 & 19.3 & 15.7 \\
\hline 1585 & 0.9000 & & 1.80 & 3.5760 & 62.1 & 36.9 & 24.7 & 21.2 \\
\hline 1995 & 0.9000 & & 1.80 & 3.5656 & 84.8 & 47.6 & 32.1 & 28.5 \\
\hline 2512 & 0.9000 & 0.5120 & 1.80 & 3.5546 & 117.7 & 61.6 & 43.0 & 37.8 \\
\hline 3162 & 0.9000 & & 1.80 & 3.5427 & 166.8 & 79.9 & 59.8 & 48.9 \\
\hline 3981 & 0.9000 & 0.544 & 1.80 & 3.5304 & 242.8 & 104.2 & 84.9 & 63.4 \\
\hline \multicolumn{9}{|c|}{ RGB - with mass loss } \\
\hline 794 & 0.8441 & 0.4002 & 1.90 & 3.6034 & 27.5 & 18.1 & 12.5 & 9.6 \\
\hline 1000 & 0.8340 & 0.41 & 1.90 & 3.5949 & 36.5 & 23.4 & 16.0 & 12.7 \\
\hline 1259 & 0.8166 & & 1.90 & 3.58 & & 30.5 & 20.6 & 17.3 \\
\hline 1585 & 0.7915 & 0.4417 & 1.90 & 3.5746 & 69.2 & 40.0 & 27.2 & 24.2 \\
\hline 1995 & 0.7679 & 0.4565 & 1.90 & 3.5629 & 98.7 & 52.7 & 37.4 & 32.7 \\
\hline 2239 & 0.7628 & 0.4640 & 1.90 & 3.5572 & 117.7 & 60.2 & 44.3 & 37.3 \\
\hline \multicolumn{9}{|c|}{ AGB - with mass loss } \\
\hline 794 & 0.7371 & 0.4737 & 1.90 & 3.6045 & 29.9 & 19.1 & 13.1 & 10.5 \\
\hline 1000 & 0.7344 & 0.4783 & 1.90 & 3.5956 & 39.9 & 24.6 & 16.7 & 14.1 \\
\hline 1259 & 0.7301 & & 1.90 & 3.5862 & 53.9 & 31.7 & 21.5 & 19.0 \\
\hline 1585 & 0.7244 & 0.4913 & 1.90 & 3.5762 & 74.0 & 40.9 & 28.5 & 25.3 \\
\hline 1995 & 0.7118 & 0.5005 & 1.90 & 3.5654 & 104.6 & 53.2 & 39.4 & 32.9 \\
\hline 2512 & 0.6903 & 0.5120 & 1.90 & 3.5539 & 154.2 & 70.0 & 56.8 & 42.8 \\
\hline 3162 & 0.6567 & 0.5265 & 1.90 & 3.5441 & 237.1 & 93.6 & 81.9 & 57.0 \\
\hline 3981 & 0.6057 & 0.5448 & 1.90 & 3.5478 & 321.2 & 123.2 & 99.9 & 74.1 \\
\hline 4217 & 0.5904 & 0.5500 & 1.90 & 3.5571 & 320.6 & 124.6 & 96.1 & 73.9 \\
\hline 4467 & 0.5743 & 0.5556 & 1.90 & 3.5789 & 291.6 & 113.7 & 81.3 & 66.7 \\
\hline 4571 & 0.5675 & 0.5579 & 1.90 & 3.6012 & 266.7 & 98.6 & 67.0 & 57.3 \\
\hline
\end{tabular}

Notes: $\ell / H_{\mathrm{p}}$ is the ratio of mixing-length to pressure scale height. $P_{0}, P_{1}, P_{2}$ and $P_{3}$ are the linear periods (in days) of the fundamental, 1 st, 2nd and 3 rd overtone modes.

\subsection{Large amplitude variables}

In contrast to the small amplitude variables, the largeamplitude variables i.e. the Miras are consistent with theoretical models without mass loss, while the models with mass loss do not fit the observed $K-\log P$ sequence. (We ignore V19 in this comparison since Fig. 3 suggests this star is a Galactic halo star rather than a 47 Tuc star.)

We believe this contradiction can be explained by the nonlinear effects in the pulsation of red giants with large amplitudes. Some preliminary nonlinear pulsation calculations made for these models show that the full-amplitude pulsation periods are considerably shorter than the linear periods due to a change in the envelope structure associated with large amplitude pulsation (see also Ya'Ari \& Tuchman 1996). An example of the change in period of one of these models, perturbed with a low amplitude and allowed to reach limiting amplitude, is shown in Fig. 7. Thus the periods of nonlinear fundamental mode models may be able to explain the periods of the Miras in 47 Tuc, although this needs further exploration. The nonlinear models will be described elsewhere.

\subsection{Evolution of pulsation mode with luminosity}

Figure 6 provides an indication of the pulsation evolution of stars evolving up the giant branch. Ignoring V19 (as noted above), it seems that stars start pulsating at $K \sim 7.7$ in the 1 st to 3 rd overtone, then evolve up to higher luminosities to $K \sim 6.9$ where the stars transit to fundamental mode pulsation. 


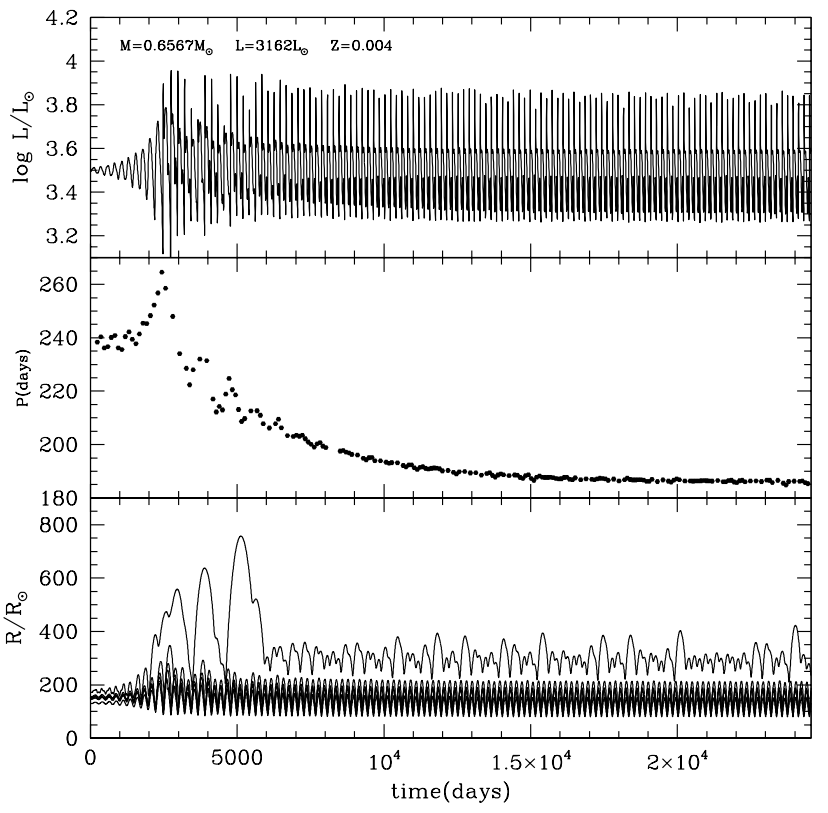

Fig. 7. Luminosity $L$, pulsation period $P$ and radii of several mass points plotted against time in a model with $M=0.6567 M_{\odot}$ and $L=3162 L_{\odot}$. The linear pulsation period of this model is 240 days while the nonlinear pulsation period after 67 years of relaxation is 185 days.

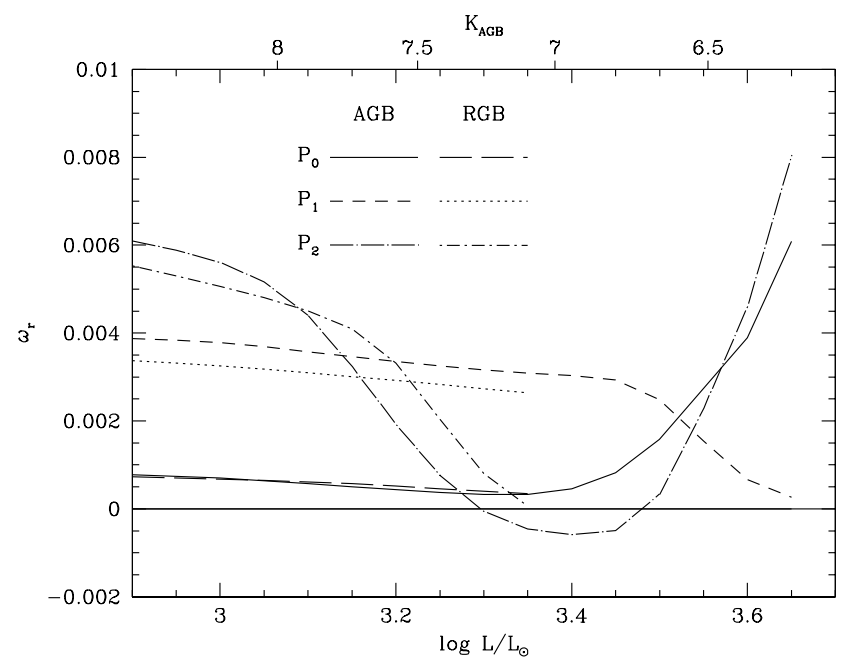

Fig. 8. The growth rate $\omega_{\mathrm{r}}\left(\right.$ days $^{-1}$ plotted against $\log L / L_{\odot}$ for the models in the mass loss sequence. $\omega_{\mathrm{r}}$ is the real part of the eigenvalue $\omega$ where a time dependence $\exp (\omega t)$ is assumed. The $K$ magnitude for AGB stars is shown on the top axis.

Further evolution is in the fundamental mode, until mass loss terminates AGB evolution at $K \sim 6.2$. This is broadly consistent with what is expected from the linear non-adiabatic growth rates of the models. These are shown plotted against luminosity in Fig. 8. It should be remembered that the theoretical growth rates for these highly convective stars are very uncertain and should be regarded as indicative only. The general behaviour shown in Fig. 8 is that, at low luminosities the 2nd overtone has the highest growth rate, at intermediate luminosities the 1 st overtone has the highest growth rate, and at high luminosities the fundamental mode grows most rapidly. The 2 nd overtone also has a high growth rate at high luminosities but it is likely to be overwhelmed by the fundamental mode in the nonlinear case. This suggests an evolution from 2 nd to 1 st overtone and then to fundamental mode. This is similar to the observed situation, although the overtones co-exist rather than following a distinct progression with luminosity.

\section{Summary and conclusions}

We have identified 22 new variable red giants in 47 Tuc and determined periods for another 8 previously known variables. All red giants redder than $V-I_{\mathrm{c}}=1.8$ are variable at the limits of our detection threshold, which corresponds to $\delta V \approx 0.1 \mathrm{mag}$. This colour limit corresponds to a luminosity $\log L / L_{\odot}=3.15$ and it is considerably below the tip of the RGB at $\log L / L_{\odot}=$ 3.35 . In the $K-\log P$ diagram, the 47 Tuc variables do not closely follow the ridge lines of PL relations seen for LPVs in the MCs, indicating that the PL relations are mass dependent.

Linear non-adiabatic modelling was used to try to reproduce the observed PL relations, especially for the low amplitude pulsators where linear calculations should be appropriate. It was shown that models without mass loss can not reproduce the observed PL laws for the low amplitude pulsators. Models that lose sufficient mass to terminate AGB evolution near $L \sim 4000 L / L_{\odot}$ do reproduce the observed PL relations for low amplitude variables. This is the first time that measurements of the masses of stars on the AGB have shown that mass loss of the order of $0.3 M_{\odot}$ occurs along the RGB and AGB.

The linear pulsation periods do not agree well with the observed periods of the large amplitude Mira variables, which pulsate in the fundamental mode. The solution to this problem appears to be that the nonlinear pulsation periods in these low mass stars are considerably shorter than the linear pulsation periods due to a rearrangement of stellar structure caused by the pulsation. Although such effects have been seen in pulsation models before, the 47 Tuc stars studied here provide the first observational evidence for this effect.

The observations show that stars evolve up the RGB and first part of the AGB pulsating in low order overtone modes, then switch to fundamental mode at high luminosities. The linear non-adiabatic growth rates of models suggest that such behaviour should occur but the models at this stage are only indicative. It is hoped that future improved models including the effect of turbulent viscosity (e.g. Olivier \& Wood 2005) will allow a reliable determination of the growth rates and mode selection processes in red giant stars, as well as an estimation of the effect of nonlinear pulsation on the pulsation period.

Acknowledgements. We are indebted to Laszlo Kiss for kindly providing additional images for our time series of 47 Tuc. We wish to thank Christophe Alard for support with the image subtraction code ISIS and for providing his PSF fitting software. We thank Brian Schmidt for organizing the monitoring of 47 Tuc at Mount Stromlo Observatory, and the queue mode observers at ESO $2.2 \mathrm{~m}$ and CTIO $1.3 \mathrm{~m}$. This project obtained data via the NOAO share of the SMARTS consortium. TL has been supported by the Austrian Academy of Science (APART programme). PRW has been partially supported by a grant from the Australian Research Council. This publication makes use of 
data products from the Two Micron All Sky Survey, which is a joint project of the University of Massachusetts and the Infrared Processing and Analysis Center/California Institute of Technology, funded by the National Aeronautics and Space Administration and the National Science Foundation.

\section{References}

Alard, C. 2000, A\&AS, 144, 363

Alexander, D. R., \& Ferguson, J. W. 1994, ApJ, 437, 879

Allen, D. A., \& Cragg, T. A. 1983, MNRAS, 203, 777

Alcock, C., Axelrod, T. S., Bennett, D. P., et al. 1992, in Robotic Telescopes in the 1990s, ed. A. V. Fillippenko, ASP Conf. Ser. 34, 193

Bessell, M. S. 1979, PASP, 91, 589

Boothroyd, A. I., \& Sackmann, I.-J. 1988, ApJ, 328, 641

Caretta, E., \& Gratton, R. G. 1997, A\&AS, 121, 95

Carpenter, J. M. 2001, AJ, 121, 2851

Clement, C. M., Muzzin, A., Dufton, Q., et al. 2001, AJ, 122, 2587

DePoy, D. L., Atwood, B., Belville, S. R., et al. 2003, SPIE, 4841, 827

Eggen, O. J. 1975, ApJ, 195, 661

Fagotto, F., Bressan, A., Bertelli, G., \& Chiosi, C. 1994, A\&AS, 105, 29

Fox, M. W. 1982, MNRAS, 199, 715

Fox, M. W., \& Wood, P. R. 1982, ApJ, 259, 198

Fraser, O. J., Hawley, S. L., Cook, K. H., \& Keller, S. C. 2005, AJ, 129,768

Frogel, J. A., Persson, S. E., \& Cohen, J. G. 1981, ApJ, 246, 842

Gratton, R. G., Bragaglia, A., Carretta, E., et al. 2003, A\&A, 408, 529

Hesser, J. E., Harris, W. E., Vandenberg, D. A., et al. 1987, PASP, 99, 739

Houdashelt, M. L., Bell, R. A., \& Sweigart, A. V. 2000a, AJ, 119, 1448

Houdashelt, M. L., Bell, R. A., Sweigart, A. V., \& Wing, R. F. 2000b, AJ, 119, 1424

Iglesias, C. A., \& Rogers, F. F. 1993, ApJ, 412, 572
Ita, Y., Tanabé, T., Matsunaga, N., et al. 2004, MNRAS, 347, 720

Kalzuny, J., Kubiak, M., Szymanski, M., et al. 1998, A\&AS, 128, 19

Kiss, L. L., Szatmary, K., Cadmus, R. R., \& Mattei, J. A. 1999, A\&A, 346,542

Landolt, A. U. 1992, AJ, 104, 340

Lebzelter, T., Wood, P. R., Hinkle, K. H., Joyce, R. R., \& Fekel, F. C. 2004, Proc. IAU Coll., 193, Variable stars in the Local Group, ASP Conf. Ser., 310, 144

Lebzelter, T., Wood, P. R., Hinkle, K. H., Joyce, R. R., \& Fekel, F. C. 2005, A\&A, 432, 207

Lloyd-Evans, T. 1974, MNRAS, 167, 393

Marshall, S. 1994, in Astronomy from wide-field imaging, ed. H. T. MacGillivray, et al. (Dordrecht: Kluwer), IAU Symp., 161, 67

Menzies, J. W., \& Whitelock, P. A. 1985, MNRAS, 212,783

Olivier, E. A., \& Wood, P. R. 2005, submitted

Origlia, L., Ferraro, F. R., Fusi Pecci, F., \& Rood, R. T. 2002, ApJ, 571,458

Percy, J. R., Bessla, G., Velocci, V., \& Henry, G. W. 2003, PASP, 115, 479

Ramdani, A., \& Jorissen, A. 2001, A\&A, 372, 85

Reimers, D. 1975, in Problems in Stellar Atmospheres and Envelopes, ed. B. Bascheck, W. H. Kegel, \& G. Traving (Springer: Berlin), 229

Schuller, F., Ganesh, S., Messineo, M., et al. 2003, A\&A, 403, 955

Soszyński, I., Udalski, A., Kubiak, M., et al. 2004, Acta Astr., 54, 129

Sperl, M. 1998, Comm. Asteroseism., 111, 1

Weldrake, D. T. F., Sackett, P. D., Bridges, T. J., \& Freeman, K. C. 2004, AJ, 128, 736

Wood, P. R., \& the MACHO Collaboration 1999, in Asymptototic Giant Branch Stars, ed. T. Le Bertre, A. Lèbre, \& C. Waelkens (San Francisco: ASP), IAU Symp., 191, 151

Wood, P. R. 2000, PASA, 17, 18

Wood, P. R., Olivier, E. A., \& Kawaler, S. D. 2004, ApJ, 604, 800

Ya'Ari, A., \& Tuchman, Y. 1996, ApJ, 456, 350 